\title{
Note on numerical study of the beam energy spread in NDCX-I
}

\author{
by \\ J.-L. Vay, P.A. Seidl, and A. Friedman
}

from

Lawrence Berkeley National Laboratory (on behalf of U.S. HIFS-VNL)

I Cyclotron Road, Berkeley, CA 94720

Accelerator Fusion Research Division

Berkeley, Califomia 94720

and

Lawrence Livermore National Laboratory

January 19,201$\}$

This work was supported by the Director, Office of Science, Office of Fusion Energy Sciences, of the U.S. Department of Energy under Contract No, DE-AC02-05CHII231. 
This document was prepared as an account of work sponsored by the United States Government. While this document is believed to contain correct information, neither the United States Government nor any agency thereof, nor The Regents of the University of Californis, nor any of their employees, makes any warranty, express or implied, or assumes any legal responsibility for the accuracy, completeness, or usefulness of any information, apparatus, product, or process disclosed, or represents that its use would not infringe privately owned rights. Reference herein to any specific commercial product, process, or service by its trade name, trademark, manufacturer, or otherwise. does not necessarily constitute or imply its endorsement, recommendation, or favoring by the United States Government ot any agency thereof, or The Regents of the University of California. The views and opinions of authors expressed herein do not necessarily state or reflect those of the United States Government or any agency thereof or The Regents of the University of California.

This work was supporled by the Directot, Office of Science, Office of Fusion Inergy Sciences, of the U.S. Department of Energy under Contact No. DE-AC02-0SCH। $123 \mathrm{I}$. 


\title{
Note on numerical study of the beam energy spread in NDCX-I
}

\author{
J.-L. Vay, P.A. Seidl, A. Friedman
}

(Dated: 19 January 2011)

The kinetic energy spread (defined here as the standard deviation of the beam particle energies) sets the ultimate theoretical limit on the longitudinal compression that can be attained on NDCX-I and NDCX-II. Experimental measurements will inevitably include the real influences on the longitudinal phase space of the beam due to injector and accelerator field imperfections ${ }^{1}$. These induced energy variations may be the real limit to the longitudinal compression in an accelerator. We report on a numerical investigation of the energy spread evolution in NDCX-I; these studies do not include all the real imperfections, but rather are intended to confirm that there are no other intrinsic mechanisms (translaminar effects, transverse-longitudinal anisotropy instability, etc.) for significant broadening of the energy distribution.

(a)

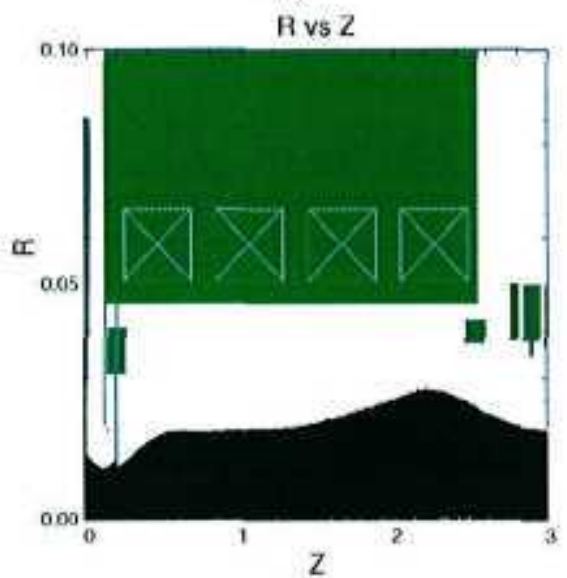

(c)

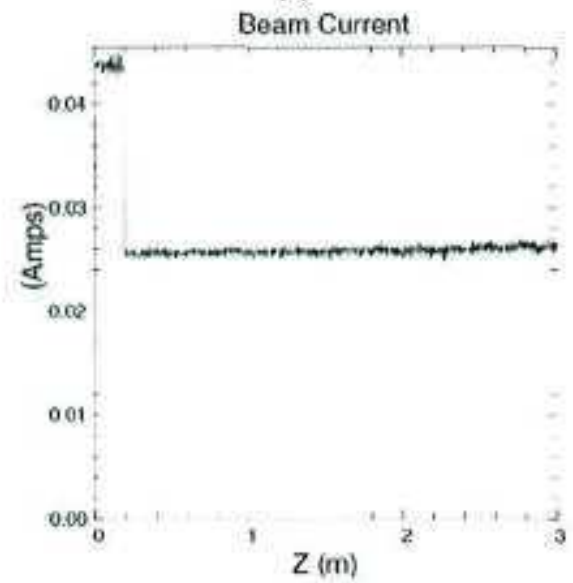

(b)

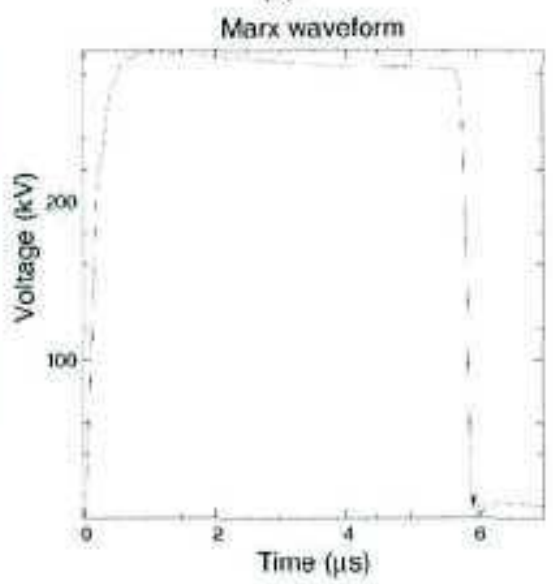

(d)

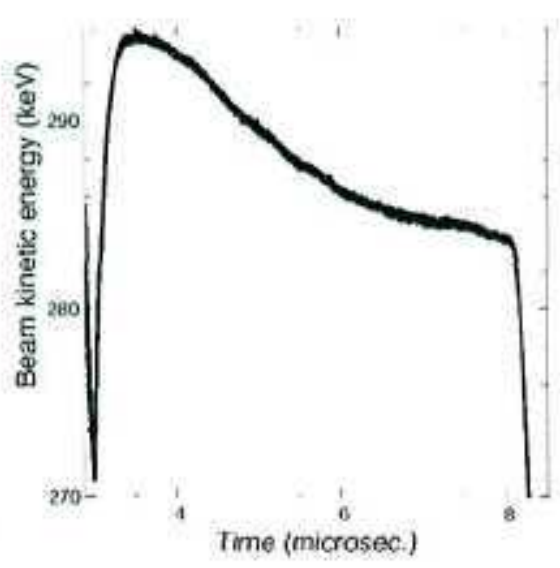

FIG. 1. From a Warp simulation of NDCX-1 (smapshots taken at $\mathrm{t}=4 \mu \mathrm{s}$ ), (a) snapshot of the beam (black) and lattice (green), (b) Marx waveform, (c) snapshot of beam current, (d) kinetic energy history by of beam macroparticles hitting the end plate at $z=3 \mathrm{~m}$.

We have performed Warp simulations that use a realistic Marx voltage waveform which was derived from experimental measurements (averaged over several shots), a fully-featured model of the accelerating and focusing lattice, and new diagnosties for computing the local energy spread (and temperature) that properly account for linear cortelations that arise from the discrete binning along each physical dimension (these capabilities reproduce and extend those of the earlier HIF code $\mathrm{BPIC}^{2}$; see Appendix I for more details). The new diagnostics allow for the calculation of multi-dimensional maps of energy spread and temperature in 2-D axisymmetric or 3-D Cartesian space at selected 
(a)

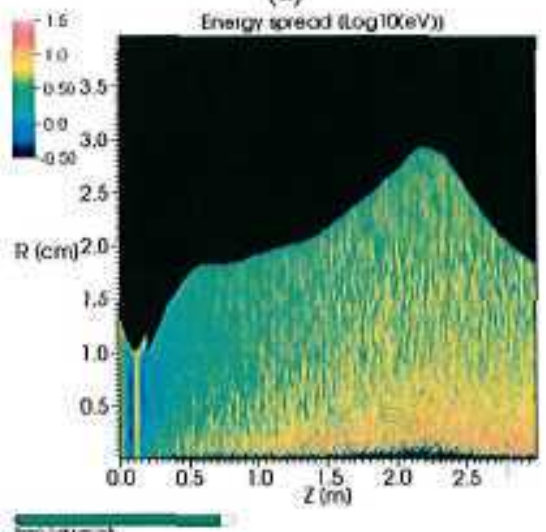

(b)

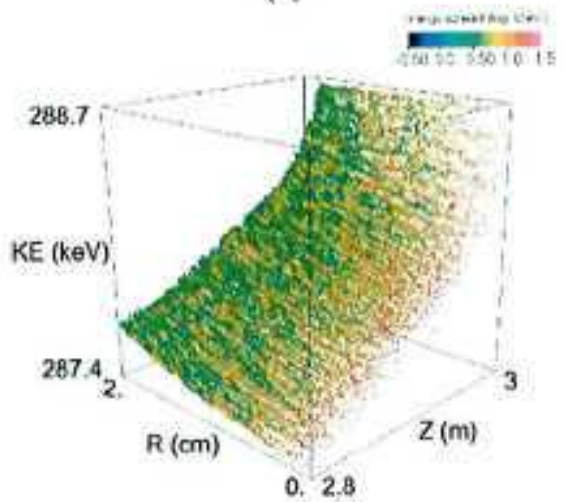

FIG. 2. From a Warp simulation of NDCX-I , (a) 2.D axisymmetric map of energy spread (in Log10(eV)); (b) scatter plot of kinetic energy versus radius and longitudinal position $(0.28 \mathrm{~m}<z<3 \mathrm{~m})$ colored according to local energy spread.
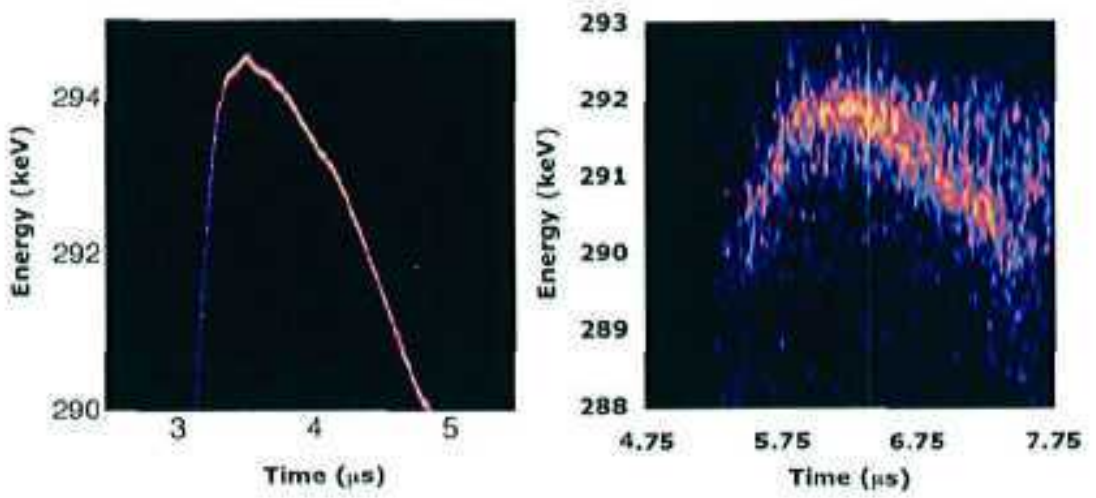

FIG. 3. NDCX-1 beam energy history from (left) Warp simulation; (right) NDCX-I measurement using a spectrometer and a streak camera.

times. The simulated beam-line was terminated at $\mathrm{z}=3 \mathrm{~m}$ by a conducting plate, so as to approximately reproduce the experimental conditions at the entrance of the spectrometer that was used for mapping the longitudinal phase space.

Snapshots of the beam projection and current, as well as the Marx waveform and history of beam kinetic energy collected at the end plate, are shown in Fig. 1. A two-dimensional axisymmetric map of energy spread from simulations of a typical NDCX-1 configuration is shown in Fig. 2 (a). The energy spread starts at $0.1 \mathrm{eV}$ at the source and rapidly rises to a few $\mathrm{eV}$, then fluctuates between a ftaction of an $\mathrm{eV}$ and tens of $\mathrm{eV}$, ending near the exit in a range of a few $\mathrm{eV}$ at the outer edge of the beam to a few tens of $\mathrm{eV}$ near the axis. The higher value on-axis is associated with greater numerical noise there, due to the axisymmetric geometry of the calculation, resulting in poorer simulation-particle statistics at small radius. A scatter plot of the macroparticles kinetic energy (KE) versus radius (R) and longitudinal position $(0.28 \mathrm{~m}<z<3 \mathrm{~m})$ colored by local energy spread is shown in Fig. 2 (b). As expected, there is a correlation of the kinetic energy with radius that is clearly visible at $z=2.8 \mathrm{~m}$ and vanishes at the metal plate at $z=3 \mathrm{~m}$. More snapshots from simulations varying the time step, grid resolution and number of macroparticles are given in Appendix II.

The macro-particles were collected at the exit plate and their kinetic energy history is plotted in Fig. 3 (left) and contrasted to an experimental measurement using a streak camera shown in Fig. 3 (right) (taken from [1]). For some types of measurements, averaging over several pulses to improve signal-to-noise will contribute an additional spread that may not be present on any single beam pulse [1]. The upper bound for the energy spread is in the range of a few $100 \mathrm{eV}$ for the experiment while in the range of a few eV for the reported Warp simulations. The Marx voltage exhibits variations in the range of up to several hundreds of volts, playing a significant role in the experimentally measured energy spread, which may account for the difference between the experimental and the simulated bounds. 


\section{APPENDIX A - CALCULATION OF THE TEMPERATURE AND ENERGY SPREAD IN WARP}

Assuming a distribution of paicteles $f(X, V)$ of posiluons $X=\left\{x_{1} y_{1} z\right\}$ and velocities $V=\left\{v_{x}, v_{y}, v_{f}\right\}$, the term-

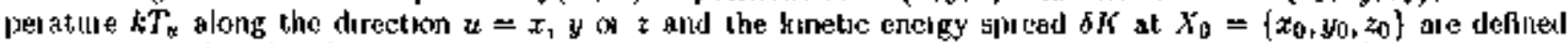
as bemg duectly related respechively to the var ince of the velocity components and the standart deviation of the kunctic estergy as

$$
\begin{aligned}
& k T_{u}=\frac{1}{2} m \overline{\left(v_{u}-\overline{v_{u}}\right)^{2}}=\frac{1}{2} m \frac{\int_{-\infty}^{\infty} \int\left(X_{0}, v_{u}\right)\left(v_{u}-\overline{v_{n}}\right)^{2} d v_{v}}{\int_{-\infty}^{\infty} \int\left(X_{0}, v_{u}\right) d v_{v}} \\
& s K=\sqrt{(K-\bar{K})^{2}}=\frac{1}{2} \sqrt{\frac{\int_{0}^{\infty} f\left(X_{0}, V\right)(K-\bar{K})^{2} d K}{\int_{0}^{\infty} \sqrt{\left(X_{0}, V\right)}}},
\end{aligned}
$$

where $m, v=\sqrt{v_{x}^{2}+v_{v}^{2}+x_{2}^{2}}$ and $K=\frac{1}{2} m^{2}$ ale lespectively the moss, velocity and kinetic encigy ol the partic|cs, and the mean valtes of the velocity conponents atd kinetuc eneigy are given by

$$
\begin{aligned}
& \overline{v_{u}}=\frac{\int_{-\infty}^{\infty} f\left(X_{0}, v_{u}\right) t_{u} d v_{u}}{\int_{-\infty}^{\infty} f\left(X_{0}, v_{u c}\right) d t_{u t}} \\
& \bar{K}=\frac{\int_{0}^{\infty} f\left(X_{0}, V\right) K d K}{\int_{0}^{\infty} \int\left(X_{0}, V\right) d K}
\end{aligned}
$$

The above definitions spoply to unfiustesumal volumes and contınwous density functions f and can be unteipreted as

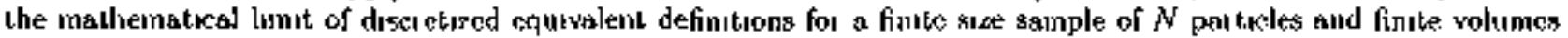
$\delta \Omega$ wher $N \rightarrow \infty$ and $\delta \Omega \rightarrow 0$ At these limits, correlations between velocity (or kinetic energy) whth position vantsit

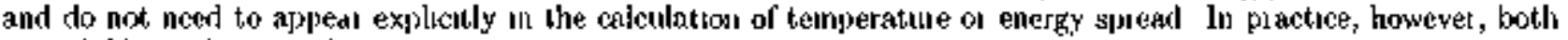
$N$ and $\delta \Omega$ are fiute, and con elations nexd eventual $y$ to be accounted for

As an example, we conyidea a hrictpriol fiom a simulation of NDCX-I The longitudinal phase space $z-v_{2}$ is plotted

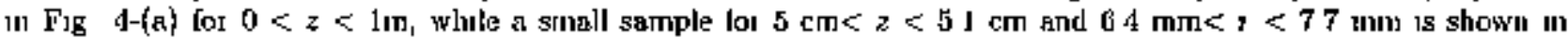
Fis 4-(h), and with averages ienoved in Fig 4-(c) A lineal conelation is clealy visible, which would give rise to

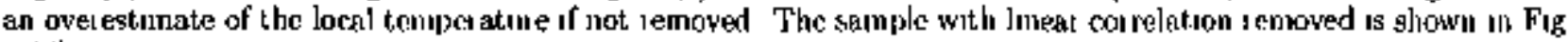
4-(d)

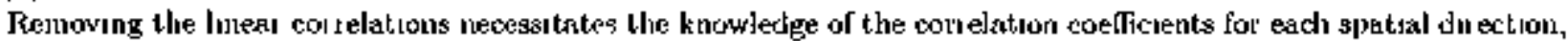
which dile compuled as follows

Let us assume that at $X_{0}=\left\{x_{0}, y_{0}, z_{0}\right\}$, the kinetic eneigy $K_{1}=K-\bar{\kappa}$ has a linear corıeladion with the

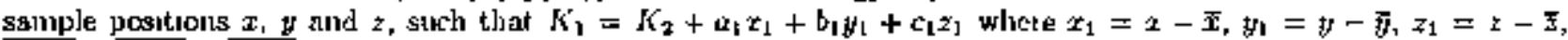
$\overline{7_{1} K_{2}}=\overline{y_{1} K_{2}}=\overline{z_{1} K_{2}}=0$, and $a_{1}$, $b_{1}$ and $c_{1}$ ale scalors giving the "slopes' of the coipling in each gpatial dirextion Fiom the defirutions givell above, one call wite

$$
\begin{aligned}
& \overline{\tau_{1} K_{2}}=\overline{x_{1}\left(\overline{\left.K_{1}-a_{1} x_{1}-b_{1} H_{1}-c_{1} z_{1}\right)}\right.}=0 \\
& \overline{y_{1} K_{2}}=\overline{y_{1}\left(K_{1}-a_{1} x_{1}-b_{1} t_{1}-c_{1} z_{1}\right)}=0 \\
& \overline{z_{1}} K_{2}=\overline{z_{1}\left(\overline{\left.K_{1}-a_{1} a_{1}-b_{1} y_{1}-c_{1} z_{1}\right)}\right.}=0
\end{aligned}
$$

such wat $a_{1}, b_{1}$ and $c_{1}$ are the sotutions of the incon syatem

$$
\left\{\begin{array}{l}
\overline{x_{1}^{2}} \alpha_{1}+\overline{x_{1} y_{1}} b_{1}+\overline{\bar{t}_{1}} c_{1}-\overline{x_{1} K_{1}}=0 \\
\overline{y_{1} x_{1}} a_{1}+\overline{\eta_{1} \bar{y}_{1}} c_{1}-\overline{y_{1} \mu_{1}}=0 \\
\overline{z_{1} x_{1}} a_{1}+\overline{z_{1} y_{1}} b_{1}+\overline{z_{1}^{2}} c_{1}-\overline{z_{1} K_{1}}=0
\end{array}\right.
$$

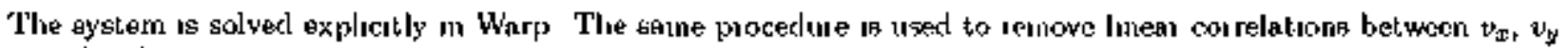
and $v_{2}$ for the temperature calculations

Althongh not presenl, (ot discenable) un the I mm sample that was shown, hugher order conelations can be

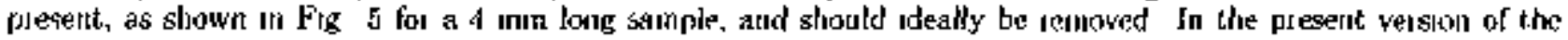

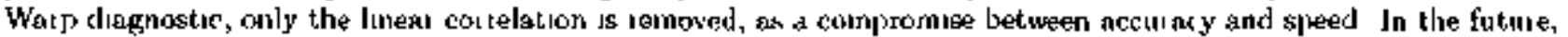

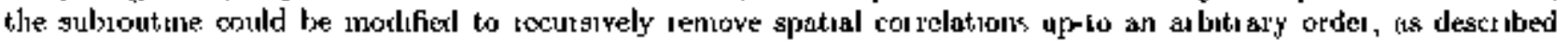
bedow 
(a)

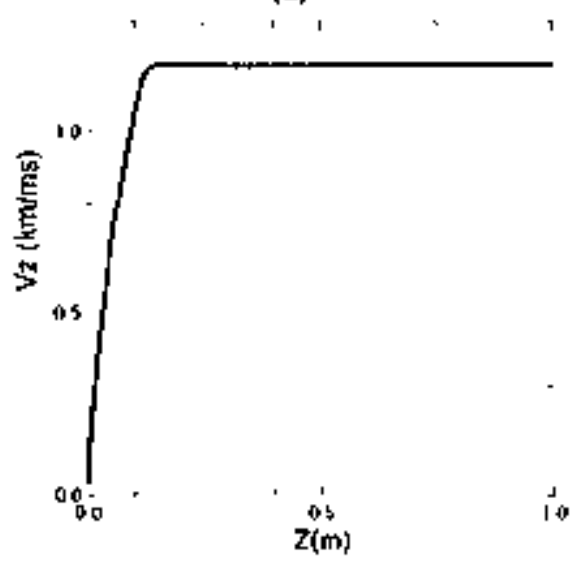

(c)

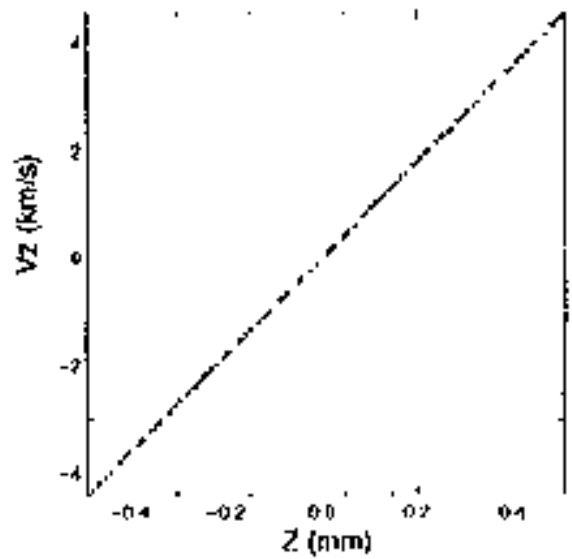

(b)

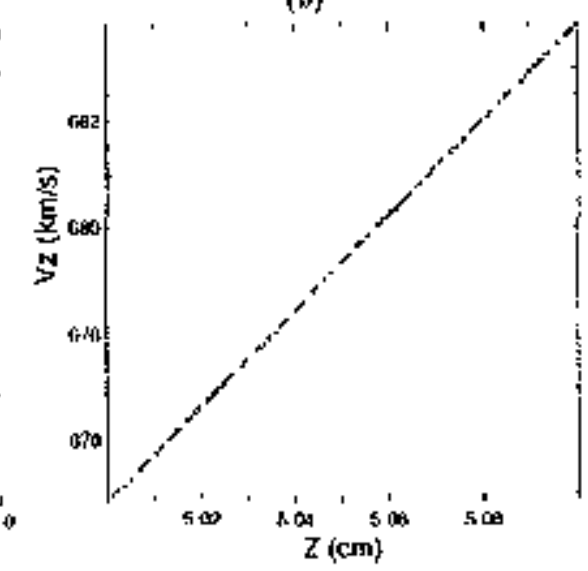

(d)

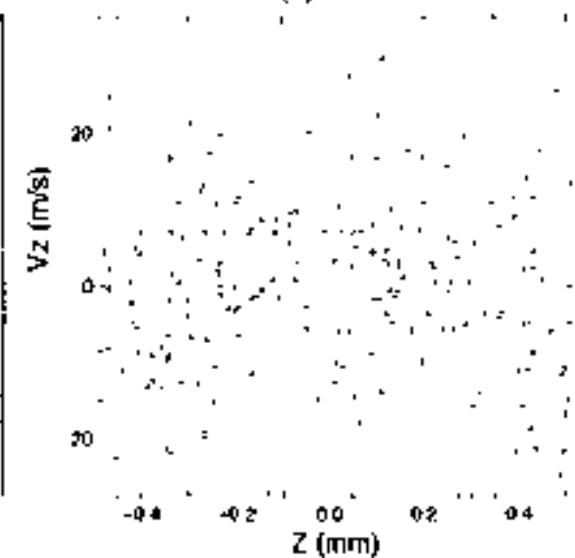

FIG. 4. Longituclinal $z-y_{4}$ phetse-space projection from a simulation of NDCX-I for (a) $0<z<1$ m, (b) a small sartiple

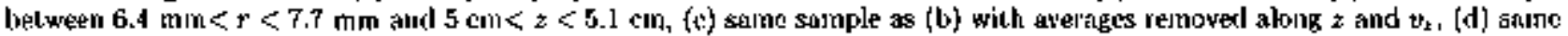
sample as (c) with linear conrrelation between $v_{2}$ ard 2 reinoved.

More gencrally, one can write the kimetic energy distribution as the weighted sum of powers of $\Sigma_{1,}$ yl and $z_{1}$ up to orkler $n$

$$
K=K_{n+1}+\sum_{i=0}^{n}\left(a_{1} \dot{r}_{1}^{i}+b_{1} y_{1}^{\prime}+c_{1} z_{1}^{i}+d_{4}\right)
$$

while imposing $\overline{x^{n} K_{n+1}}=\overline{y^{n} K_{n+1}}=\overline{z^{n} K_{n+1}}=0$ and $\overline{K_{n}}=0$. The distribution $K_{n+1}$ can be computed recursively using

$$
K_{\mathrm{n}+1}=K_{n}-a_{n} x_{1}^{k}-b_{n} y_{1}^{n}-c_{n} z_{1}^{\prime \prime}-d_{n}
$$

and solving for

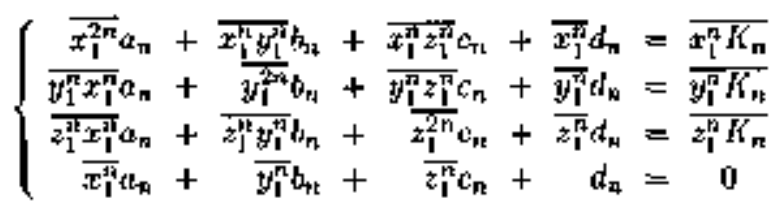

We note that for $n$ odd, then $\overline{x_{1}^{*}}=\overline{y_{1}^{77}}=\overline{z_{1}^{\pi}}=0$, giving $d_{t_{1}}=0$ and the system to solve simplifies to

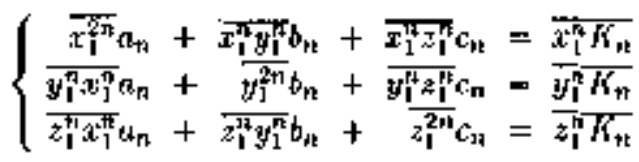


(a)

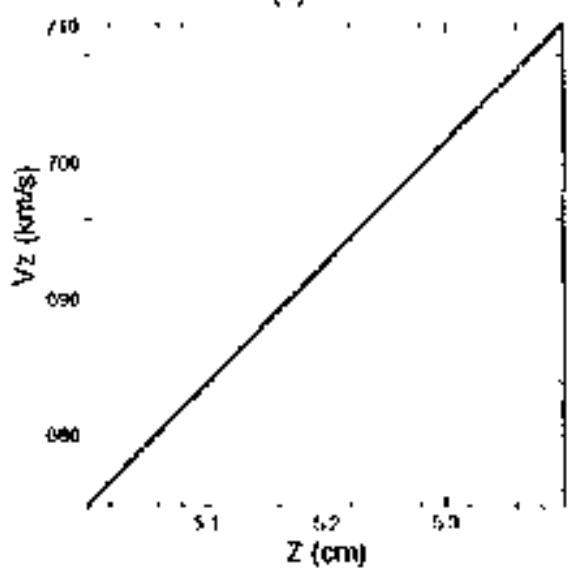

(c)

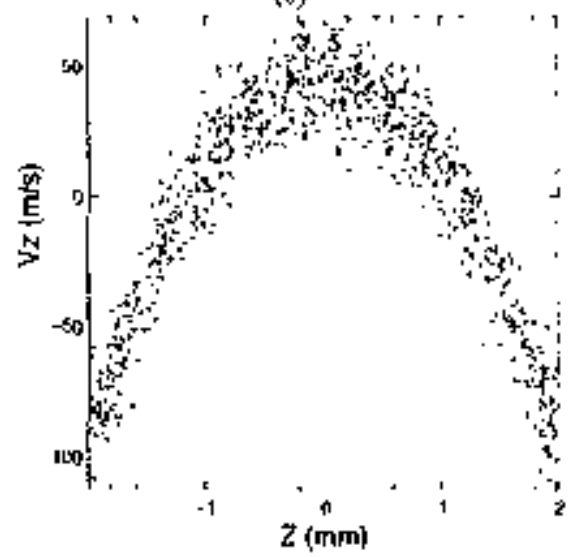

(b)

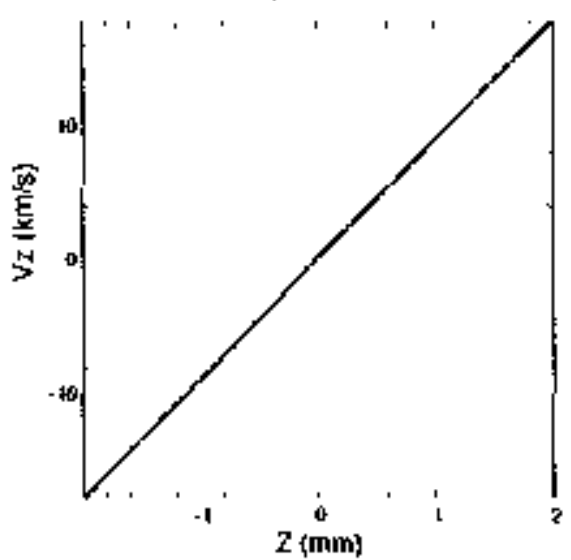

(11)

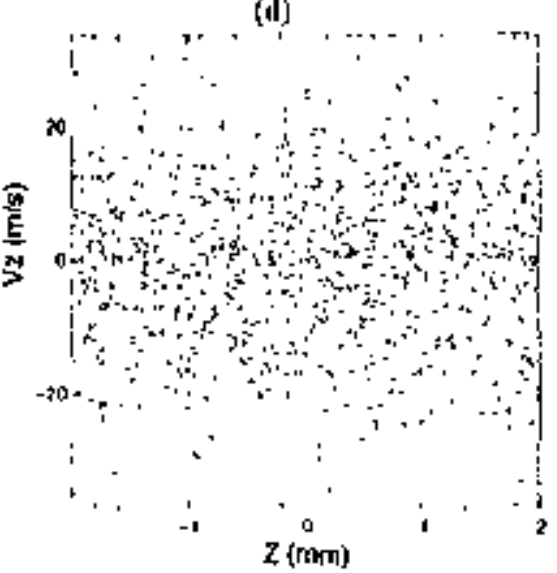

FIG. 5. longitudinal $z-v_{x}$ phase.space projection from a sinulation of NDCX.I lor (a) a amall sample between 6.4 m tru< $y<7.7$ Irım and $5 \mathrm{cre}<z<5.4 \mathrm{~cm}$, (b) sane sample as (a) with averages removed along 2 and $v_{2}$, (c) sanie saniple as (b) witll linear correlation between $v_{s}$ and $z$ remowed (d) aame semple es (c) with quadralic currelaton between $t$. and $z$ removed.

The recuryive procedure thal was just deseribed has been applied suocessfully to the sample case shown in lijg. 5 , for removing the qutadratic correlation between $v_{z}$ and $z$ that is visible in Fijg. 5 (c). giving the uncorrelated distribution ploted in Flg. 5-(d). Comparing Fig. 4-(d) and 5-(d) showi that renoving higher swder cotrelations allows for larger samples, reducing the size of the diagnostic arrays and jinproving the statistic per cell. This procedure will be implemented in Watp in the next luture, adting the option of either sctling a maximum bevel of rectursion level (or coluivalently highesl order of correlation to be removed) or settimg a tolerance for stopping the recursion on the difterence between the temperatore (or energy spread) value at two consecutive rescursion lewels. Sorting the macroparticle data by temperature (or energy spread) gricl cells beforehand would allow for cacli cell to rench inelependent levels of tecursion.

Finally, although this was nou discussed here, the exnitter regiot presents a nout sirngularity which calls for finer sampling of the diagnostic. Tluss, accurate and delailed calculation of energy spread and temperature near the source would benefit from adaptive mesh refinenrent (AMIR) of the diagnostic grid near thase singular l'egions. The AMR tools developed for field calcudations may dilus be of beneft for thise diagriustics.

\section{APPENDIX B - WARP SIMULATIONS OF NDCX-I VARYING NUMERICAL PARAMETERS}

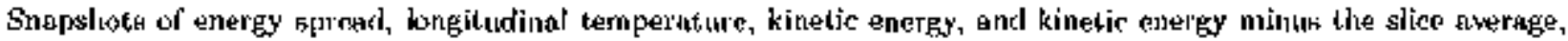
are plottod Iron Warp simulations of NDCX-I for yarious nunerical parameters:

- Fig. 6: $\delta x=\delta z=2 \mathrm{~mm}, \delta t=1.25 \mathrm{~ns}, N_{p} \approx 1.8$ inillion inacroparticles, 
- Fig. $7: \delta x=\delta z=2 \mathrm{~mm}, \delta t=0.625 \mathrm{~ns}, N_{p} \approx 1.8$ million macroparticles,

- Fig. $8: \delta x=0.4 \mathrm{~mm}, \delta z=1 \mathrm{~mm}, \delta t=1.25 \mathrm{~ns}, N_{p} \approx 1.8$ million macroparticles,

- Fig. 9: $\delta x=\delta z=2 \mathrm{~mm}, \delta t=1.25 \mathrm{~ns}, N_{p} \approx 18$ million macroparticles,

- Fig. 10: $\delta x=0.4 \mathrm{~mm}, \delta z=1 \mathrm{~mm}, \delta t=1.25 \mathrm{~ns}, N_{p} \approx 18$ million macroparticles.

From the limited set of numerical parameters that were involved, the following indications emerge: the energy spread and longitudinal temperature are not sensitive to the time step but are very sensitive to the resolution and the number of macroparticles. This suggests that numerical noise is contributing to unphysical heating and that the simulation with the highest resolution and number of macroparticles may not yet be converged. The usage of standard techniques for noise reduction (e.g. higher order particle shapes, filtering of charge density) should be considered for future simulations, with the caveat that care must be exercised near conductors (this may need some study and algorithm development). We finally note that despite the fact that convergence was not demonstrated on the highest resolution run, the upper bound that was obtained for energy spread is well below the one that has been obtained experimentally, and is thus deemed adequate for the present purpose.
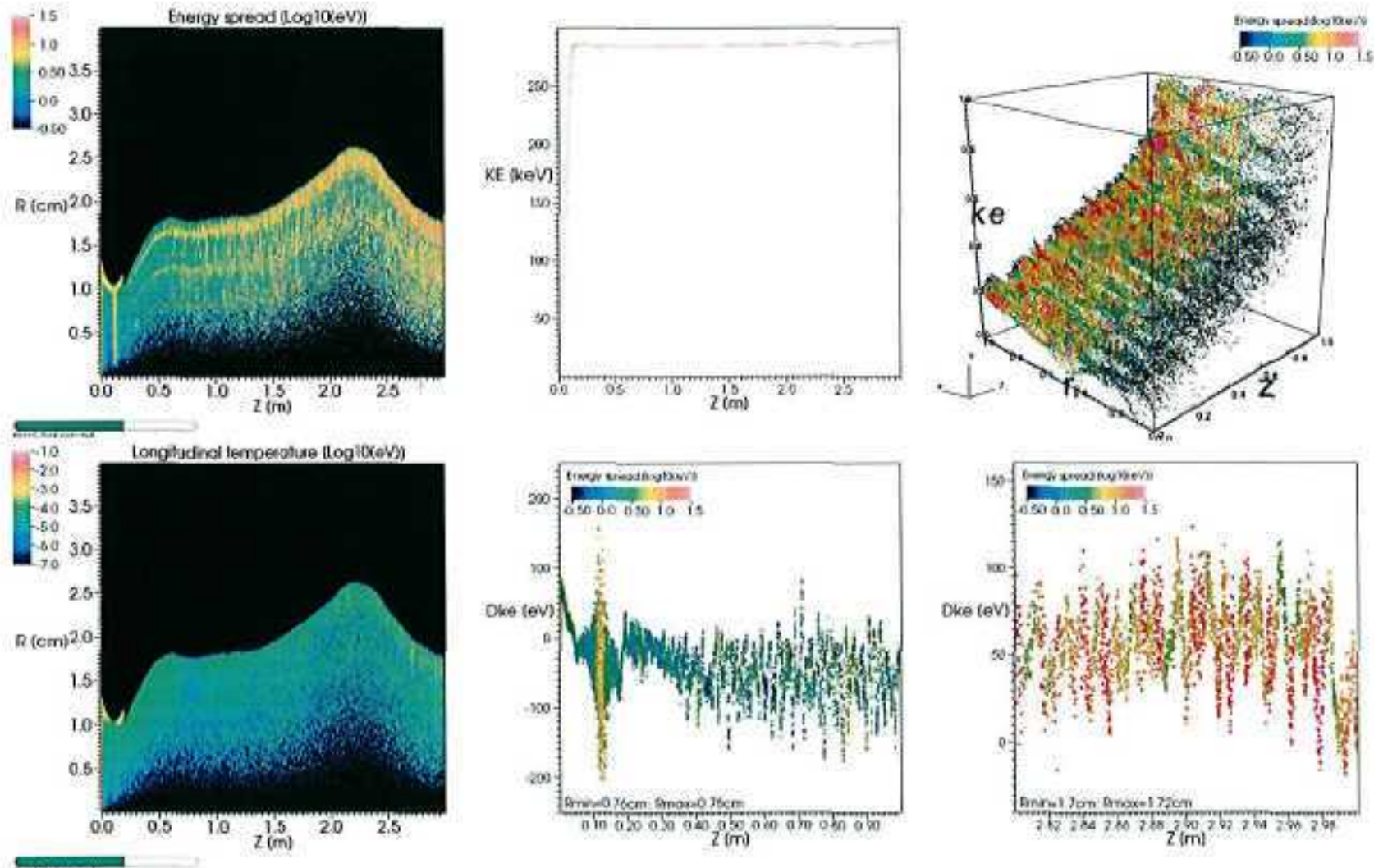

FIG. 6. From Warp simulation of NDCX-I with $\delta x=\delta z=2 \mathrm{~mm}, \delta t=1.25 \mathrm{~ns}, N_{p} \approx 1.8$ million macroparticles: snapshots of (top-left) beam kinetic energy spread vs $(r, z)$; (top-tniddle) kinetic energy vs $z$; (top-right) kinetic energy vz $(r, z)$, colored by energy spread for $2.8 \mathrm{~m}<z<3 \mathrm{~m}$; (botiom-left) longitudinal temperature $\mathrm{vs}(r, z)$; (bottom-middle) kinetic energy minus averaged stice energy for $0<z<1 \mathrm{~m}$ and a thin annulus $0.76 \mathrm{~cm}<r<0.78 \mathrm{~cm}$, colored by energy spread; (bottom-right) same as (bottom-middle) but for $2.8 \mathrm{~m}<z<3 \mathrm{~m}$ and $1.7 \mathrm{~cm}<\mathrm{r}<1.72 \mathrm{~cm}$. 

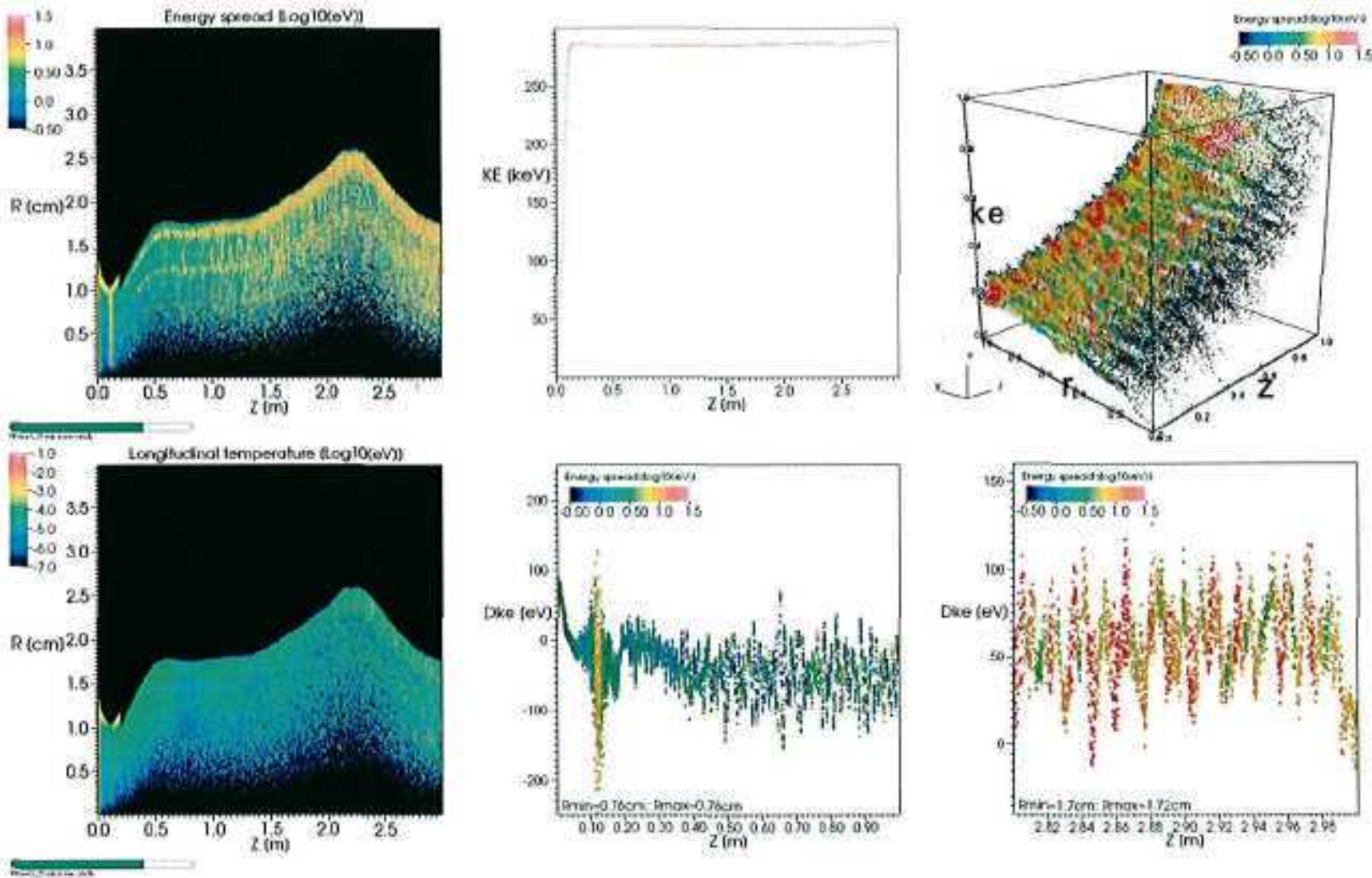

FIG. 7. From Warp simulation of NDCX-I with $\delta x=\delta z=2 \mathrm{~mm}, \delta t=0.625 \mathrm{~ns}, N_{p} \approx 1.8$ million macroparticles: snapshots of (top-left) beam kinetic energy spread vs $(r, z)$; (top-middle) kinotic energy vs z; (top-right) kinetic energy vz ( $r, z)$, colored by energy spread for $2.8 \mathrm{~m}<z<3 \mathrm{~m}$; (bottom-left) longitudinal temperature vs $(r, z)$; (bottom-middle) kinetic energy minus averaged slice energy for $0<z<1 \mathrm{~m}$ and a thin annulus $0.76 \mathrm{~cm}<r<0.78 \mathrm{~cm}$, colored by energy spread; (bottom-right) same as (bottom-middle) but for $2.8 \mathrm{~m}<z<3 \mathrm{~m}$ and $1.7 \mathrm{~cm}<x<1.72 \mathrm{~cm}$. 

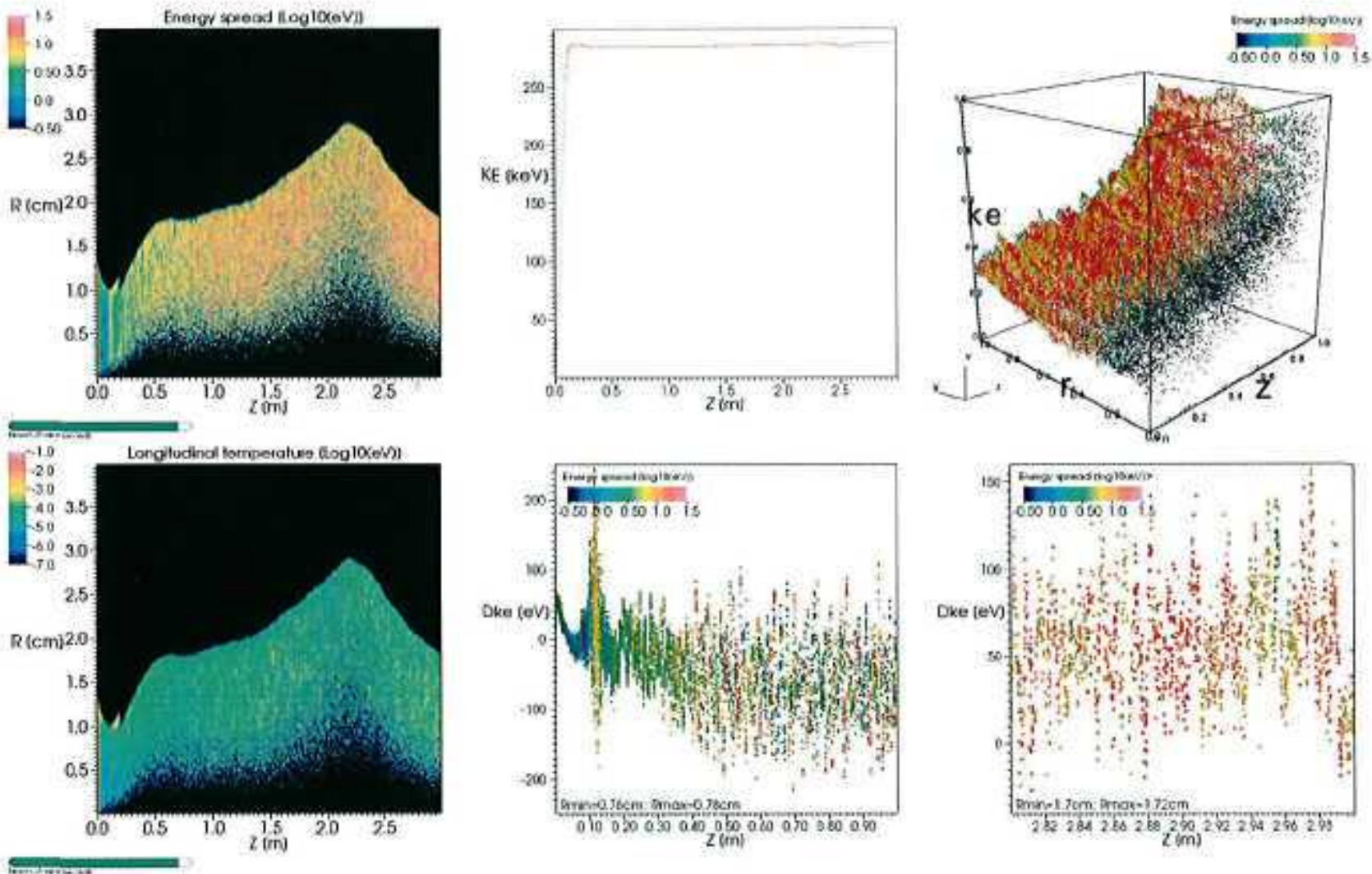

FIG 8 . From Warp simulation of NDCX-I with $\delta x=0.4 \mathrm{~mm}, \delta z=1 \mathrm{~mm}, \delta t=1.25 \mathrm{~ns}, N_{p} \approx 1.8$ million macroparticles: snapshots of (top-left) beam kinetic energy spread vs $(r, z)$; (top-middle) kinetic energy vs $z$; (top-right) kinetic energy vz $(r, z)$, colored by energy spread for $2.8 \mathrm{~m}<z<3 \mathrm{~m}$; (bottom-left) longitudinal temperature vs $(r, z)$; (bottom-middle) kinetic energy minus averaged slice energy for $0<z<1 \mathrm{~m}$ and a thin annulus $0.76 \mathrm{~cm}<\mathrm{r}<0.78 \mathrm{~cm}$, colored by energy spread; (bottom-right) same as (bottom-middle) but for $2.8 \mathrm{~m}<z<3 \mathrm{~m}$ and $1.7 \mathrm{~cm}<r<1.72 \mathrm{~cm}$. 

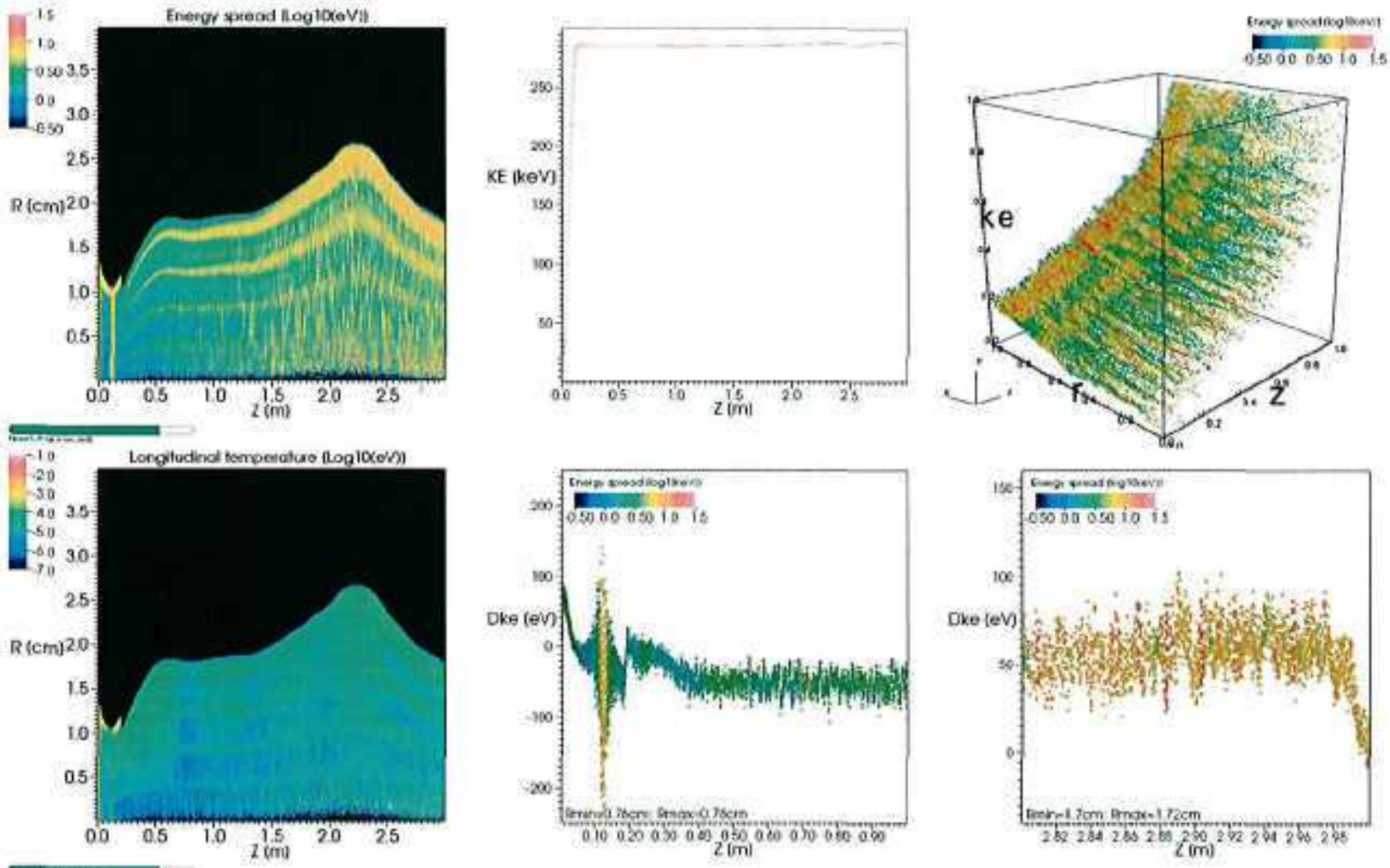

FIG. 9. From Warp simulation of NDCX-1 with $\delta x=\delta z=2 \mathrm{~mm}, \delta t=1.25 \mathrm{~ns}, N_{\mathrm{p}} \approx 18$ million macroparticles: snapshots of (top-left) beam kinetic energy spread vs $(r, z)$; (top-middle) kinetic energy vs $z$; (top-right) kinetic energy vz $(r, z)$, colored by energy spread for $2.8 \mathrm{~m}<z<3 \mathrm{~m}$; (bottom-left) longitudinal temperature vs $(r, z)$; (bottom-middle) kinetic energy minus averaged slice energy for $0<z<1 \mathrm{~m}$ and a thin annulus $0.76 \mathrm{~cm}<r<0.78 \mathrm{~cm}$, colored by energy spread; (bottom-right) same as (bottom-middle) but for $2.8 \mathrm{~m}<z<3 \mathrm{~m}$ and $1.7 \mathrm{~cm}<r<1.72 \mathrm{~cm}$. 

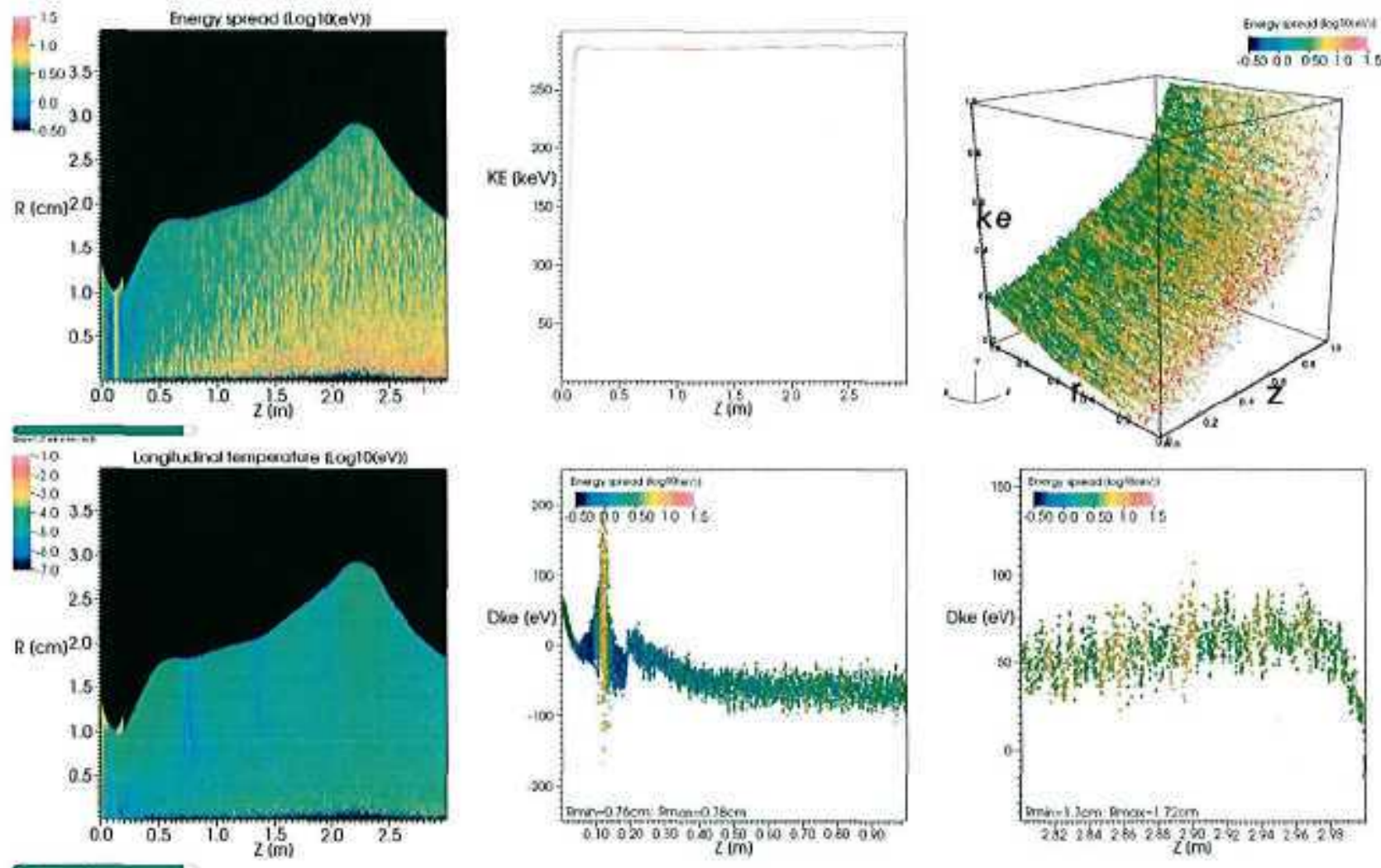

FIG. 10. From Warp simulation of NDCX-I with $\delta x=0.4 \mathrm{~mm}, \delta z=1 \mathrm{~mm}, \delta t=1.25 \mathrm{~ns}, N_{p} \approx 18$ million macroparticles: snapshots of (top-left) beam kinetic energy spread vs $(r, z)$; (top-middle) kinetic energy vs $z$; (top-right) kinetic energy vz $(r, z)$, colored by energy spread for $2.8 \mathrm{~m}<z<3 \mathrm{~m}$; (bottom-left) longitudinal temperature vs $(r, z)$; (bottom-middle) kinetic energy minus averaged slice energy for $0<z<1 \mathrm{~m}$ and a thin annulus $0.76 \mathrm{~cm}<r<0.78 \mathrm{~cm}$, colored by energy spread; (bottom-right) same as (bottom-middle) but for $2.8 \mathrm{~m}<z<3 \mathrm{~m}$ and $1.7 \mathrm{~cm}<r<1.72 \mathrm{~cm}$. 